\title{
Sodium Valproate in the Treatment of Sydenham's Chorea
}

Sir,

Sydenham's chorea is a movement disorder with no universally accepted effective drug therapy for its rapid control. We conducted this prospective study to validate efficacy and safety of sodium valproate's in it. The study was conducted over a period of 4 years in the department of Pediatrics, NRS Medical College, Kolkata. The diagnosis was clinical after exclusion of all other known causes of chorea. Ten patients fulfilled the inclusion criterion ( 7 girls and 3 boys with mean age of 9.7 years. They were given sodium valproate at a dose of $20 \mathrm{mg} /$ $\mathrm{kg} /$ day in 2 or 3 divided doses. If any patient was already on any drug for the treatment of chorea, it was stopped for a week and valproate therapy was started thereafter. A blinded observer assessed all the children daily till they were discharged from the hospital. Response to treatment was assessed clinically. 'Recovery' was defined as total absence of chorea. Any other clinical improvement short of total absence of chorea, patients responding within 96 hours of commencement of therapy were considered as "incomplete recovery" and 'rapid responders' respectively . Treatment was continued for 12 weeks in all patients who responded to valproate therapy and was withdrawn gradually over a week. Follow up of the patients was done for one year to see for relapse of chorea and to monitor for any side effect.

All the patients were from low socioeconomic class. Duration of illness ranged from two weeks to one year. All had generalized chorea. In eight children, chorea was the only mode of presentation. Associated polyarthritis and symptomatic carditis were observed in one case each. All cases had ASO titer less than 200 i.u except in one case. Abnormality in mitral valve was detected by $2 \mathrm{D}$ Echocardiography in 5 cases. As far as the cardiac function was concerned all were asymptomatic except one with carditis. All were newly diagnosed cases of chorea and had received some form of treatment other than valproate before entering in present trial. All of them received phenobarbitone except one who received both pheobarbitone and haloperidol. Duration of therapy before entering in present study was from 3 days to 2 months.

Four children responded within $72 \mathrm{hrs,} \mathrm{four} \mathrm{responded}$ within $96 \mathrm{hrs}$ and the remaining two responded within 7 days of commencement of therapy. Thus, eight $(8 / 10)$ patients were 'rapid responders'. 'Recovery' occurred within
7 days of commencement of therapy in 6 patients and all the patients were chorea free within 2 weeks of commencement of therapy. Valproate was continued for a period of 12 weeks in all children. The dose of valproate required no change. During the one year follow up neither relapse nor any side effect was observed.

The pathogenesis of Sydenham's chorea is not clearly known and is probably an autoimmune response of the central nervous system to group A streptococci resulting in functional overactivity of the dopaminergic system ${ }^{1}$ Sodium valproate acts via its effect on GABA to inhibit the supposedly dopaminergic overactivity and thus controls involuntary movements. Occasional relapse and partial response to valproate therapy has been observed. ${ }^{2}$ Majority $(8 / 10)$ of our patients were rapid responders and there was no treatment failure or relapse. This may be due to continuation therapy for a longer period, irrespective of rapidity of response and the use of high dose of valproate right from the initiation of therapy, in comparison to previous studies where therapy was started with $15 \mathrm{mg} / \mathrm{kg} /$ day and increased to $25 \mathrm{mg} /$ $\mathrm{kg} /$ day if poor response was observed..$^{2-5}$ Therefore, sodium valproate appears to be an effective and safe drug for the treatment of Sydenham's chorea.

\section{Tapas K. Sabui and Pragya Pant Department of Pediatrics, Nilratan Sircar Medical College, 138, AJC Bose Road, Kolkata 700014, India. E-mail: tsabui@gmail.com [DOI-10.1007/s12098-010-0074-5]}

\section{REFERENCES}

1. Naidu S, Narasimhachari N. Sydenham's chorea: A possible presynaptic dopaminergic dysfunction initially. Ann Neurol 1980; 8: 445-447.

2. Daoud AS, Zaki M, Shakir R, al-Saleh Q. Effectiveness of sodium valproate in the treatment of Sydenham's chorea. Neurology 1990; 40: 1140-1141.

3. Dhanraj M, Radhakrishnan AR, Srinivas K, Sayeed ZA. Sodium valproate in sydenham's chorea. Neurology 1985; 35: 114-115.

4. Davutoglu V, Kilinc M, Dinckal H et al. Sydenham's choreaclinical characteristics of nine patients. Int J Cardiol 2004; 96: 483-484.

5. Alvarez LA, Novak G. Valproic acid in the treatment of Sydenham's chorea. Pediatr Neurol 1985; 1: 317-319. 